\title{
Apple Peel Biochemical Changes after Foliar Application of Combined Boron and Calcium I. Phenolics and Physico-Chemical Attributes
}

\author{
A. Mwije1,2, E. W. Hoffman ${ }^{2,3}$, E. Lötze ${ }^{2 *}$ \\ ${ }^{1}$ Makerere University, Kampala, Uganda \\ ${ }^{2}$ University of Stellenbosch, Stellenbosch, South Africa \\ ${ }^{3}$ University of Queensland, Brisbane, Australia \\ Email: ^elotze@sun.ac.za
}

How to cite this paper: Mwije, A., Hoffman, E.W. and Lötze, E. (2020) Apple Peel Biochemical Changes after Foliar Application of Combined Boron and Calcium I. Phenolics and Physico-Chemical Attributes. American Journal of Plant Sciences, 11, 965-986. https://doi.org/10.4236/ajps.2020.116069

Received: May 11, 2020

Accepted: June 27, 2020

Published: June 30, 2020

Copyright $\odot 2020$ by author(s) and Scientific Research Publishing Inc. This work is licensed under the Creative Commons Attribution International License (CC BY 4.0).

http://creativecommons.org/licenses/by/4.0/ (c) (i) Open Access

\begin{abstract}
Post-full bloom foliar applications of boron in combination with calcium $(\mathrm{B}+\mathrm{Ca})$ suppress fruit sunburn-browning incidence in apples (Malus domestica). However, the mode-of-action is not yet explained and hence, protocols to exploit this seemingly cost-effective peel physiology augmenting approach compared to usual methods (shade-netting, overhead cooling and kaolin application which attenuate excess fruit heat-load), cannot be fully developed or promoted justifiably among growers. Apple fruit sunburn-browning disorders are rampant worldwide, partly because of climate change as well as high cost and inefficacy of the established methods. However, knowledge of $\mathrm{B}+\mathrm{Ca}$ effect on apple peel physiology may unravel the mode-of-action, paving the way for commercialization of this novel approach. In this study, B+Ca effect on apple peel phenolics and selected fruit physico-chemical attributes was investigated, using studies conducted at two farms for two seasons in Western Cape, South Africa. Four foliar treatments varying in B, Ca and inclusion of zinc ( $\mathrm{Zn}$ ), were applied on 'Cripps Pink', 'Golden Delicious' and 'Granny Smith' apples in 2014/15 (only 'Cripps Pink') and 2015/16 (all cultivars) in randomized complete block design experiments with five replications. Fruit peel samples for quantitative determination of total phenolics and total flavonoids analyses were collected at different fruit maturity stages. Significant $(p<0.05)$ interaction (treatments and fruit maturity stages) effect was observed for total phenolics ('Golden Delicious' and 'Granny Smith' only) and total flavonoids (all cultivars). Physico-chemical attributes (fruit diameter, size and total soluble solids) were only evaluated in 'Golden Delicious' and 'Granny Smith', and no significant differences among treatments occurred. Since no clear treatment differences in apple peel phenolic patterns were ob-
\end{abstract}


served, other appropriate biochemical aspects like photosynthetic pigments should be investigated among such foliar $\mathrm{B}+\mathrm{Ca}$ treatments.

\section{Keywords}

Flavonoids, Malus domestica, Sunburn

\section{Introduction}

Post-full-bloom foliar boron in combination with calcium $(\mathrm{B}+\mathrm{Ca})$ has shown potential as an alternative approach to mitigate sunburn-browning incidence in apple orchards [1] [2] [3] [4], but its underlying mode-of-action is not yet elucidated. The lack of a scientific explanation of the underlying mode-of-action has impeded efforts to refine application protocols for on-farm use as well as commercialization of this envisaged low-cost approach in mitigating the rampant apple fruit sunburn-browning disorders especially amongst large scale growers. A study on tree physiological, fruit anatomical and peel mineral concentration changes in 'Cripps Pink' and 'Golden Delicious' apples treated with foliar B+Ca in which some treatments suppressed class 1 (mild sunburn-browning) incidence, failed to reveal the mode-of-action [4]. This initiated the hypothesis of a physiological mode-of-action induced by foliar $\mathrm{B}+\mathrm{Ca}$ treatments through augmentation of levels of relevant fruit peel biochemical aspects [5] [6]. Therefore, studies on the physiological effect of foliar $\mathrm{B}+\mathrm{Ca}$ on apple peel biochemistry could possibly lead to the unravelling of the sought mode-of-action.

Plant tissue B nutrition status influences respective phenolic levels as well as affecting cellular functions [7] [8] [9] [10]. The application of kaolin (contains calcium oxide) increased phenolic levels in Vitis vinifera L. grapes reportedly through a positive effect on the phenylpropanoid and flavonoid pathways [11]. Regimens of foliar Ca nutrition and peel flavonoids showed positive correlation in 'Elstar' apples [12]. Two applications of a Phostrade-Ca formulation at five and three weeks before commercial harvest increased peel phenolic levels in 'Braeburn' apples [13]. Phostrade Ca is concentrated liquid phosphorous that contains as well $\mathrm{Ca}$ and nitrogen and is used as foliar application in apple orchards to improve fruit quality [13]. On the other hand, apple peel phenolic levels increase during sunburn-browning development ostensibly as an innate resistance mechanism [14] [15]. An increase in 'Braeburn' apple peel phenolics prevented photodamage and subsequently sunburn [16], and [17] reported a similar effect of phenolics accumulation limiting 'skin burning' in 'Cameo' apple.

Following the above findings in plant tissues, nutritional supplementation of $\mathrm{B}$ and $\mathrm{Ca}$ to apple fruit through foliar $\mathrm{B}+\mathrm{Ca}$ could possibly augment phenolic biochemical levels in peel tissues. In order to address this critical knowledge gap, the objective of this study was to determine the effect of varying B and Ca concentrations and inclusion or not of Zinc (Zn) in specific post-full-bloom foliar 
$\mathrm{B}+\mathrm{Ca}$ treatments on three phenolic attributes (total phenolics, total flavonoids and total flavonoids to total phenolics ratio) and three fruit physico-chemical attributes (fruit size, diameter and total soluble solids). This was performed in three different apple cultivars popularly grown in Western Cape, South Africa.

\section{Materials and Methods}

\subsection{Study Sites and Experiments Design}

Apple cultivars 'Cripps Pink', 'Golden Delicious', and 'Granny Smith', were used in randomized complete block design experiments on two commercial apple farms in Western Cape, South Africa. These experimental farms were, 1) Applethwaite (Pty) farm ( $34^{\circ} 12^{\prime} 08.0^{\prime} \mathrm{S}$; $18^{\circ} 59^{\prime} 16.5^{\prime} \mathrm{E}$ ) in Elgin ('Golden Delicious' and 'Granny Smith') and 2) Welgevallen Experimental Farm of Stellenbosch University ( $\left.33^{\circ} 56^{\prime} 52.5^{\prime} \mathrm{S} ; 18^{\circ} 52^{\prime} 19.9^{\prime} \mathrm{E}\right)$ in Stellenbosch ('Cripps Pink'). The experiments had five replications, using single trees as experimental units. Apple trees were trained as central leaders on M793 rootstock and received fertilizer application, pest and disease control, thinning and irrigation according to commercial practice. The experiment with 'Cripps Pink' was consecutively conducted in both 2014/15 and 2015/16 seasons, whereas 'Golden Delicious' and 'Granny Smith' experiments were only conducted in 2015/16.

\subsection{Formulation of Treatments}

Formulation of treatments and choice of materials followed indications obtained in previous findings from [1] with regard to combining B with Ca for successful suppression of sunburn-browning in 'Golden Delicious' apples. In 2014/15, three treatments were used, 1) B'0.00+Ca'0.00 (plain water with no added $\mathrm{B}+\mathrm{Ca}$ ), 2) B’0.02+Ca’0.10 (6 ml Manni-Plex ${ }^{\circledast} \mathrm{B}$ plus $10 \mathrm{ml} \mathrm{Manni-Plex}{ }^{\circledast} \mathrm{Ca}$ ), and 3) B’0.02+Ca’1.29 (6 ml Manni-Plex $\mathrm{B}^{\circledast}$ plus $68 \mathrm{ml} \mathrm{YaraLiva}{ }^{\mathrm{Tm}}$ Calcinit $^{\mathrm{TM}}$ ). Treatment codes as used represent B and Ca levels as weight/weight according to respective formulation product labels, as guided by composition of products as indicated in Table 1. For instance, B’0.02+Ca'0.10 infers $0.02 \mathrm{~g} \cdot \mathrm{l}^{-1} \mathrm{~B}$ plus $0.10 \mathrm{~g} \cdot \mathrm{l}^{-1}$ Ca which were tank-mixed in $10 \mathrm{~L}$ of water. All treatment formulation products used in 2014/15 were sourced from Elim Kunsmis (Pty) Ltd., South Africa, except Calcinit $^{\mathrm{Tm}}$ sourced from Yara Business Unit Africa, South Africa. In 2014/15, 'Cripps Pink' full-bloom occurred on 13 October 2014, and foliar treatments started at 28 DAFB (11 November 2014), continuing at weekly intervals respectively on 18, 25, November 2014 and 1, 8, 17 December 2014.

In 2015/16, treatments were, 1) B'0.00+Ca'0.00, 2) B'0.02+Ca'0.06+Zn'0.03 (6 ml Manni-Plex ${ }^{\circledast}$ B plus 10 ml Manni-Plex ${ }^{\circledast}$ Cal-Zn), 3) B’0.02+Ca’1.24 (6 ml Manni-Plex ${ }^{\circledast}$ B plus 65 g Calsol $^{\oplus}$ ). Treatment 4) B’0.08+Ca’1.24 (5 g Spraybor ${ }^{\circledast}$ plus $65 \mathrm{~g} \mathrm{Calsol}^{\circledR}$ ), whereas 5), B’0.17+Ca’1.24 (10 $\mathrm{g} \mathrm{Spraybor}^{\varpi}$ plus $65 \mathrm{~g} \mathrm{Calsol}^{\circledR}$ ). All treatment formulation products used in 2015/16 were sourced from NexusAG (Pty) Ltd., South Africa, except Spraybor ${ }^{\circledast}$ which was obtained from Nulandis (Pty) Ltd., South Africa. In 2015/16, 'Cripps Pink' full-bloom occurred 
Table 1. Composition of the products used to formulate foliar treatments.

\begin{tabular}{cc}
\hline Trade name & Active Ingredients \\
\hline Manni-Plex ${ }^{\circ} \mathrm{Ca}$ & $10 \% \mathrm{Ca}, 8 \% \mathrm{~N}$ \\
Manni-Plex Cal-Zn & $6 \% \mathrm{Ca}, 6 \% \mathrm{~N}, 3 \% \mathrm{Zn}$ \\
Manni-Plex ${ }^{\circ}$ & $3.3 \% \mathrm{~B}, 5 \% \mathrm{~N}$ \\
YaraLiva $^{\mathrm{mat}} \mathrm{Calcinit}^{\mathrm{Tax}}\left(\mathrm{Ca}\left(\mathrm{NO}_{3}\right)_{2}\right)$ & $19 \% \mathrm{Ca}, 26.3 \% \mathrm{CaO}, 15.5 \% \mathrm{~N}$ \\
Calsol $^{\circ}\left(5 \mathrm{Ca}\left(\mathrm{NO}_{3}\right)_{2} \mathrm{NH}_{4} \mathrm{NO}_{3} \cdot 10 \mathrm{H}_{2} \mathrm{O}\right)$ & $19 \% \mathrm{Ca}, 15.5 \% \mathrm{~N}$ \\
Spraybor $^{\circ}\left(\mathrm{Na}_{2} \mathrm{~B}_{4} \mathrm{O}_{7} \cdot 10 \mathrm{H}_{2} \mathrm{O}\right)$ & $16.5 \% \mathrm{~B}$ \\
\hline
\end{tabular}

on 5 October 2015, foliar treatments began on 5 November 2015 (30 DAFB), continuing consecutively at weekly intervals on 12, 19, 27 November 2015 and 3, 10 December 2015. Both 'Golden Delicious' and 'Granny Smith' full-bloom occurred on 16 October 2015, and foliar treatments began on 25 November 2015 (39 DAFB), continuing consecutively at weekly intervals on 2, 9, 15, 22 and 29 December 2015. In all experiments, foliar treatments were applied until run-off (high volume) using motorized Stihl ${ }^{\circledR}$ backpack sprayer, targeting the developing fruitlets.

\subsection{Fruit Sampling and Sample Preparation}

At selected fruit maturity stages/days after full bloom (DAFB), four to six fruit were randomly picked from the work-row side of the trees for each treatment replicate and transported in a well-insulated cool box to the laboratory where the whole fruit was peeled to obtain peels. In 2014/15, fruit sampling occurred at four (35, 49, 56 and $70 \mathrm{DAFB})$ early fruit maturity stages, in addition, fruit was also sampled at optimum (184 DAFB) and late (214) harvest maturity dates. And, at optimum and late harvest dates, fruit was also sampled from different positions of the tree canopy (inner and outer) as well as orientation (East or West side of the tree). Because of findings from the preliminary analysis of $2014 / 15$ results, a bi-weekly interval was adopted in 2015/16, fruit sampling was maintained for both sides of the tree rows but only with outer tree canopy, and four fruit maturity stages towards harvest maturity were taken for each cultivar. In 'Cripps Pink', fruit was sampled at 122, 136, 150 and 164 DAFB, in 'Golden Delicious' at 80, 94, 108 and 122 DAFB, whereas in 'Granny Smith' fruit sampling was done at 94, 108, 122 and 136 DAFB. Fruit samples of 'Golden Delicious' and 'Cripps Pink' were peeled immediately at arrival in the laboratory, while 'Granny Smith' samples were held for one day at $-0.5{ }^{\circ} \mathrm{C}$ and peeled the next day due to limitations in skilled personnel to handle the large volume of fruit samples from the three cultivars in a single day. After fruit peeling, the peels were milled in liquid $\mathrm{N}$, and stored at $-80{ }^{\circ} \mathrm{C}$ until a quantitative determination of total phenolics and total flavonoids was performed.

\subsection{Quantitative Determination of Apple Peel Phenolics}

Apple peel phenolic levels from fruit sampled as fruit maturity progressed were 
determined using analytical grade solvents and chemicals (Ethanol (96\%) (EtOH), Folin-Ciocalteu phenol (FC) reagent, Gallic acid, Catechin, Aluminum chloride $\left(\mathrm{AlCl}_{3}\right)$, Sodium nitrite $\left(\mathrm{NaNO}_{2}\right)$, Sodium hydroxide $(\mathrm{NaOH})$, and Sodium carbonate $\left.\left(\mathrm{Na}_{2} \mathrm{CO}_{3}\right)\right)$ all sourced from Sigma Aldrich, South Africa. The Milli-Q system (Millipore, Bedford, MA, USA), was the source of double distilled water $\left(\mathrm{ddH}_{2} \mathrm{O}\right)$ as required, and a UV-visible light spectrophotometer (Cary 50 Bio, Varian, Australia (Pty) Ltd, Melbourne, Australia), was used for all absorbance measurements. All centrifugation procedures at $3220 \mathrm{~g}$ and $20000 \mathrm{~g}$ were carried out at $4^{\circ} \mathrm{C}$ using Eppendorf $\mathrm{f}^{\mathrm{m}}$ 5810R and Eppendorf ${ }^{\mathrm{m}}$ 5417R cooling centrifuges respectively.

The total phenolics was determined with slight modification of the Folin-Ciocalteu method of [18], where $100 \mathrm{mg}$ frozen sample was stirred at $4^{\circ} \mathrm{C}$ for 1 hour in darkness with $10 \mathrm{ml}$ of $80 \% \mathrm{EtOH}$ in a $50 \mathrm{ml}$ centrifuge tube. Then the mixture was centrifuged for 15 minutes at $3220 \mathrm{~g}$ and $2 \mathrm{ml}$ of supernatant was further centrifuged at $20000 \mathrm{~g}$ for 15 minutes, from which $1 \mathrm{ml}$ of resultant supernatant was collected for total phenolics quantification by adding $450 \mu \mathrm{l}$ of FC reagent (1:9 dilution with $\mathrm{ddH}_{2} \mathrm{O}$ ) to $50 \mu \mathrm{l}$ of the sample or blank ( $\left.80 \% \mathrm{EtOH}\right)$, vortexed and held for 5 minutes. Thereafter $500 \mu \mathrm{l}$ of $5.6 \% \mathrm{Na}_{2} \mathrm{CO}_{3}$ was added, vortexed and held for 90 minutes at room temperature before absorbance was measured immediately at $750 \mathrm{~nm}$. Total phenolics was the expressed in Gallic Acid Equivalents (GAE) as mg GAE.100 $\mathrm{g}^{-1}$ of peel fresh weight. Gallic Acid Equivalents were obtained from standard curve prepared with Gallic Acid which ranged from 0.05 to $0.25 \mathrm{mg} \cdot \mathrm{ml}^{-1}$.

The total flavonoids was determined with slight modification of the $\mathrm{AlCl}_{3} \mathrm{CO}-$ lorimetric method of [19], whereby $100 \mathrm{mg}$ of frozen sample was stirred at $4^{\circ} \mathrm{C}$ for 1 hour in darkness with $10 \mathrm{ml}$ of $80 \% \mathrm{EtOH}$ in a $50 \mathrm{ml}$ centrifuge tube, and followed by centrifugation at $3220 \mathrm{~g}$ for 15 minutes. Then, $2 \mathrm{ml}$ of the supernatant was centrifuged further at $20000 \mathrm{~g}$ for 15 minutes and $1 \mathrm{ml}$ of resultant supernatant was obtained for total flavonoids quantification whereby $1250 \mu \mathrm{l}$ of $\mathrm{ddH}_{2} \mathrm{O}$ was added to $250 \mu \mathrm{l}$ sample or blank (80\% EtOH), followed by addition of $75 \mu \mathrm{l}$ of $5 \% \mathrm{NaNO}_{2}$ solution, and the mixture held for 5 minutes before adding $150 \mu \mathrm{l}$ of $10 \% \mathrm{AlCl}_{3}$ and further held for 6 minutes, before $500 \mu \mathrm{l}$ of $1 \mathrm{M}$ $\mathrm{NaOH}$ and $275 \mu \mathrm{l}$ of $\mathrm{ddH}_{2} \mathrm{O}$ was added with each addition followed by vortexing. Then, absorbance was immediately measured at $510 \mathrm{~nm}$. Total flavonoids was expressed in Catechin Equivalents (CE) as mg CE. $100 \mathrm{~g}^{-1}$ of peel fresh weight. Catechin Equivalents were obtained from standard curve prepared using Catechin which ranged from 0.05 to $0.25 \mathrm{mg} \cdot \mathrm{ml}^{-1}$.

\subsection{Measurement of Fruit Physico-Chemical Attributes}

Fruit mass ( $\mathrm{g}$ ) and diameter ( $\mathrm{mm}$ ) measured using Fruit Texture Analyzer Model GS-20 fitted with scale model SK-03 for $3 \mathrm{Kg}$ and model SZ-100 for 12 to 100 mm measurements (Guss Manufacturing (Pty) limited, Strand, South Africa), and total soluble solids ( ${ }^{\circ}$ Brix, Near-Infra Red spectrometer Sun Forest Model 
H-100C, S/N HC15041P011, Korea), were determined in 'Golden Delicious' and 'Granny Smith' apples immediately after harvest as per treatment replicates.

\subsection{Statistical Analyses}

The total phenolics and total flavonoids data was used to compute a total flavonoids to total phenolics ratio and this was expressed in percentage. Then, means of the data obtained of total phenolics, total flavonoids and total flavonoids to total phenolics ratio and the three physico-chemical attributes were subjected to analysis of variance with Statistica software [20]. Differences were deemed significant for $p<0.05$, and where appropriate, means were separated using Fisher's LSD posthoc test.

\section{Results}

\section{1. 'Cripps Pink' Apples}

\subsubsection{4/15 Experiment}

Treatment and interaction $\left(B+C a^{*} D A F B\right)$ effects for the phenolic aspects studied were not significant, but DAFB effect was significant for all the three phenolic aspects as fruit maturity progressed (Table 2 ). Generally, all the phenolic parameters were lowest and not different at both 35 and 70 DAFB, and highest values were recorded at both 49 and $56 \mathrm{DAFB}$. The total phenolics means recorded at 49 and 70 DAFB were not different, whereas both total flavonoids and total flavonoids to total phenolics ratio values at 56 DAFB were significantly different from those at 49 DAFB. Only total flavonoids to total phenolics ratio values showed indifferent means between 35 and 49 DAFB. Overall, phenolic values rose significantly from 35 to 56 DAFB and at 70 DAFB reduced to similar levels recorded at $35 \mathrm{DAFB}$.

At 184 DAFB (optimum maturity), treatment, fruit orientation and interaction $\left(\mathrm{B}+\mathrm{Ca}^{*} \mathrm{O}\right)$ effects were not significant, except fruit orientation for only total flavonoids (Table 3 ), and $\mathrm{B}+\mathrm{Ca}^{*} \mathrm{O}$ for total flavonoids to total phenolics ratio means (Table 4). From Table 3, East fruit showed higher and significantly different total flavonoids than West fruit. Again, Table 4 results revealed that West fruit generally had low total flavonoids to total phenolics ratio means than East fruit except fruit under treatment $\mathrm{B}^{\prime} 0.02+\mathrm{Ca}^{\prime} 0.10$. Treatment B'0.02+Ca'0.10 showed highest fruit total flavonoids to total phenolics ratio in West fruit that was significantly different from all other values except from that of treatment B'0.02+Ca'1.29 for East fruit. The East fruit was not different across all treatments, as was the west fruit of both the control and treatment B'0.02+Ca'1.29.

At 214 DAFB (late harvest maturity), no significant treatment, fruit orientation and interaction effect occurred (Table 5). Fruit position (inward or outward of tree canopy), showed significant effect on all the three phenolic parameters, and outward fruit had highest and significantly different total phenolics and total flavonoids means compared to the inward fruit, yet the reverse was recorded with the total flavonoids to total phenolics ratio means. 
Table 2. 'Cripps Pink' apple peel phenolics (2014/15).

\begin{tabular}{cccc}
\hline Source & $\mathrm{TP}^{1}$ & $\mathrm{TF}^{2}$ & $\mathrm{TF}: \mathrm{TP}(\%)$ \\
\hline Treatments (B+Ca) & & & \\
B'0.00+Ca'0.00 & $465.65^{\mathrm{ns}}$ & $265.14^{\mathrm{ns}}$ & $56.53^{\mathrm{ns}}$ \\
B'0.02+Ca'0.10 & 445.43 & 241.11 & 54.00 \\
B'0.02+Ca'1.29 & 455.14 & 258.01 & 57.27 \\
$p$ & 0.8439 & 0.2674 & 0.1618 \\
Days after full bloom (DAFB) & & & \\
35 & $399.70^{\mathrm{b}}$ & $205.17^{\mathrm{c}}$ & $51.81^{\mathrm{b}}$ \\
49 & $504.02^{\mathrm{a}}$ & $260.59^{\mathrm{b}}$ & $52.50^{\mathrm{b}}$ \\
56 & $524.11^{\mathrm{a}}$ & $358.64^{\mathrm{a}}$ & $68.87^{\mathrm{a}}$ \\
70 & $393.78^{\mathrm{b}}$ & $194.62^{\mathrm{c}}$ & $50.55^{\mathrm{b}}$ \\
$p$ & 0.0018 & 0.0000 & 0.0000 \\
$p\left(\mathrm{~B}+\mathrm{Ca}^{*} \mathrm{DAFB}\right)$ & 0.4540 & 0.4190 & 0.1661 \\
\hline
\end{tabular}

$\mathrm{TP}=$ Total Phenolics, ${ }^{1} \mathrm{mg}$ GAE. $100 \mathrm{~g}^{-1}$ peel fresh weight and TF $=$ Total Flavonoids, ${ }^{2} \mathrm{mg}$ CE. $100 \mathrm{~g}^{-1}$ peel fresh weight.

Table 3. 'Cripps Pink' apple peel phenolics at 184 DAFB (2014/15).

\begin{tabular}{ccc}
\hline Source & $\mathrm{TP}^{1}$ & $\mathrm{TF}^{2}$ \\
\hline Treatments $(\mathrm{B}+\mathrm{Ca})$ & $547.73^{\mathrm{ns}}$ & $274.09^{\mathrm{ns}}$ \\
B'0.00+Ca'0.00 & 496.93 & 253.41 \\
B'0.02+Ca'0.10 & 516.19 & 256.03 \\
B'0.02+Ca'1.29 & 0.5560 & 0.6000 \\
$p$ & & \\
Fruit orientation $(\mathrm{O})$ & $481.53^{\mathrm{ns}}$ & $240.49^{\mathrm{b}}$ \\
West & 559.04 & $281.87^{\mathrm{a}}$ \\
East & 0.0620 & 0.0380 \\
$p$ & 0.2280 & 0.2050 \\
$p\left(\mathrm{~B}+\mathrm{Ca}{ }^{\star} \mathrm{O}\right)$ & &
\end{tabular}

TP = Total Phenolics, ${ }^{1} \mathrm{mg}$ GAE. $100 \mathrm{~g}^{-1}$ peel fresh weight and TF $=$ Total Flavonoids, ${ }^{2} \mathrm{mg}$ CE. $100 \mathrm{~g}^{-1}$ peel fresh weight.

Table 4. 'Cripps Pink' apple peel TF:TP (\%) at 184 DAFB (2014/15).

\begin{tabular}{ccc}
\hline Treatments $(\mathrm{B}+\mathrm{Ca})$ & \multicolumn{2}{c}{ Fruit orientation $(\mathrm{O})$} \\
\hline B'0.00+Ca'0.00 & West & East \\
\cline { 2 - 3 } B'0.02+Ca'0.10 & $48.67^{\mathrm{c}}$ & $51.00^{\mathrm{b}}$ \\
B'0.02+Ca'1.29 & $54.38^{\mathrm{a}}$ & $48.76^{\mathrm{bc}}$ \\
\hline
\end{tabular}

$p\left(\mathrm{~B}+\mathrm{Ca}^{\star} \mathrm{O}\right)=0.0050$. 
Table 5. 'Cripps Pink' apple peel phenolics at 214 DAFB (2014/15).

\begin{tabular}{|c|c|c|c|}
\hline Source & $\mathrm{TP}^{1}$ & $\mathrm{TF}^{2}$ & TF:TP (\%) \\
\hline \multicolumn{4}{|l|}{ Treatments $(\mathrm{B}+\mathrm{Ca})$} \\
\hline $\mathrm{B}^{\prime} 0.00+\mathrm{Ca}^{\prime} 0.00$ & $361.39^{\text {ns }}$ & $198.53^{\text {ns }}$ & $60.42^{\text {ns }}$ \\
\hline $\mathrm{B}^{\prime} 0.02+\mathrm{Ca}^{\prime} 0.10$ & 328.69 & 182.35 & 63.44 \\
\hline $\mathrm{B}^{\prime} 0.02+\mathrm{Ca}^{\prime} 1.29$ & 294.64 & 177.83 & 70.39 \\
\hline$p$ & 0.6340 & 0.8110 & 0.3130 \\
\hline \multicolumn{4}{|l|}{ Fruit position $(\mathrm{P})$} \\
\hline Inward & $171.81^{\mathrm{b}}$ & $114.74^{\mathrm{b}}$ & $73.38^{\mathrm{a}}$ \\
\hline Outward & $484.68^{\mathrm{a}}$ & $257.73^{\mathrm{a}}$ & $56.12^{\mathrm{b}}$ \\
\hline$p$ & 0.0000 & 0.0000 & 0.0040 \\
\hline \multicolumn{4}{|c|}{ Fruit orientation $(\mathrm{O})$} \\
\hline West & $283.83^{\text {ns }}$ & $160.70^{\mathrm{ns}}$ & $62.78^{\text {ns }}$ \\
\hline East & 372.65 & 211.77 & 66.72 \\
\hline$p$ & 0.1290 & 0.0740 & 0.4690 \\
\hline$p\left(\mathrm{~B}+\mathrm{Ca}^{*} \mathrm{P}\right)$ & 0.8980 & 0.8770 & 0.8330 \\
\hline$p\left(\mathrm{~B}+\mathrm{Ca}^{\star} \mathrm{O}\right)$ & 0.3280 & 0.4990 & 0.8900 \\
\hline$p\left(\mathrm{P}^{\star} \mathrm{O}\right)$ & 0.1450 & 0.1430 & 0.0580 \\
\hline$p\left(\mathrm{~B}+\mathrm{Ca}^{\star} \mathrm{P}^{\star} \mathrm{O}\right)$ & 0.3920 & 0.4910 & 0.4780 \\
\hline
\end{tabular}

$\mathrm{TP}=$ Total Phenolics, ${ }^{1} \mathrm{mg}$ GAE. $100 \mathrm{~g}^{-1}$ peel fresh weight and TF $=$ Total Flavonoids, ${ }^{2} \mathrm{mg}$ CE. $100 \mathrm{~g}^{-1}$ peel fresh weight.

\subsubsection{5/16 Experiment}

Treatments, and the interaction $\left(\mathrm{B}+\mathrm{Ca}{ }^{*} \mathrm{DAFB}\right)$ did not significantly affect total phenolics and total flavonoids to total phenolics ratio means, but only DAFB did (Table 6). And, a significant $B+C a^{*} D A F B$ effect was recorded for only total flavonoids (Table 7). With fruit maturity stages (DAFB), the highest total phenolics was at 122 DAFB which was significantly different from means recorded at all other DAFB periods (Table 6), and although total phenolics at 136 and 150 DAFB was similar, overall total phenolics decreased as fruit maturity progressed. The reverse was true for total flavonoids to total phenolics ratio means, where means increased significantly at $122 \mathrm{DAFB}$, peaked at $150 \mathrm{DAFB}$, and decreased towards 164 DAFB.

The significant $\mathrm{B}+\mathrm{Ca}^{\star} \mathrm{DAFB}$ (interaction) effect on total flavonoids means as presented in Table 7 showed that the neither the control (no $\mathrm{B}+\mathrm{Ca}$ ) nor the foliar treatments showed significant differences at any maturity stage. However, at $122 \mathrm{DAFB}$, only treatments $\mathrm{B}^{\prime} 0.17+\mathrm{Ca}^{\prime} 1.24$ (low total flavonoids) and B'0.08+Ca'1.24 (high total flavonoids) differed significantly and the same was for only treatments B'0.02+Ca'1.24 (high total flavonoids) and B'0.17+Ca'1.24 (low total flavonoids) at 136 DAFB.

As fruit maturity progressed, the control and B'0.02+Ca' 1.24 did not show 
Table 6. 'Cripps Pink' apple peel phenolics (2015/16).

\begin{tabular}{ccc}
\hline Source & $\mathrm{TP}^{1}$ & TF:TP (\%) \\
\hline Treatments (B+Ca) & $307.75^{\mathrm{ns}}$ & $45.20^{\mathrm{ns}}$ \\
B'0.00+Ca'0.00 & 294.85 & 44.10 \\
B'0.02+Ca'1.24 & 296.10 & 46.65 \\
B'0.02+Ca'0.06+Zn'0.03 & 283.65 & 46.90 \\
B'0.08+Ca'1.24 & 256.15 & 44.25 \\
B'0.17+Ca'1.24 & 0.2876 & 0.6520 \\
$p$ & & \\
Days after full bloom (DAFB) & $357.32^{\mathrm{a}}$ & $34.68^{\mathrm{d}}$ \\
122 & $302.04^{\mathrm{b}}$ & $48.52^{\mathrm{b}}$ \\
136 & $296.12^{\mathrm{b}}$ & $56.96^{\mathrm{a}}$ \\
150 & $195.32^{\mathrm{c}}$ & $41.52^{\mathrm{c}}$ \\
164 & 0.0000 & 0.0000 \\
$p$ & 0.1222 & 0.1822 \\
$p\left(\mathrm{~B}+\mathrm{Ca}^{*} \mathrm{DAFB}\right)$ & & \\
\hline
\end{tabular}

TP = Total Phenolics, ${ }^{1} \mathrm{mg}$ GAE. $100 \mathrm{~g}^{-1}$ peel fresh weight, TF:TP = Total flavonoids to total phenolics ratio.

Table 7. 'Cripps Pink' apple peel total flavonoids² (2015/16).

\begin{tabular}{ccccc}
\hline Treatments (B+Ca) & \multicolumn{3}{c}{ Days after full bloom (DAFB) } \\
B'0.00+Ca'0.00 & 122 & 136 & 150 & 164 \\
\cline { 2 - 4 } B'0.02+Ca'1.24 & $121^{\text {abcde }}$ & $147^{\text {abcde }}$ & $174^{\text {abcde }}$ & $106^{\text {abcde }}$ \\
B'0.02+Ca'0.06+Zn'0.03 & $130^{\text {abcde }}$ & $152^{\mathrm{ab}}$ & $155^{\mathrm{abcd}}$ & $76^{\text {bcde }}$ \\
B'0.08+Ca'1.24 & $153^{\text {abcde }}$ & $147^{\mathrm{abc}}$ & $172^{\mathrm{ab}}$ & $69^{\mathrm{e}}$ \\
B'0.17+Ca'1.24 & $130^{\mathrm{ab}}$ & $182^{\mathrm{abcd}}$ & $149^{\mathrm{abc}}$ & $66^{\mathrm{e}}$ \\
\hline
\end{tabular}

${ }^{2} \mathrm{mg}$ CE. $100 \mathrm{~g}^{-1}$ peel fresh weight, $p\left(\mathrm{~B}+\mathrm{Ca}^{\star} \mathrm{O}\right)=0.0030$.

significant differences in total flavonoids means, whereas only treatments B'0.02+Ca'0.06+Zn'0.03 and B'0.08+Ca'1.24 showed significantly lower total flavonoids at 164 DAFB than that at all preceding DAFB periods. Only in B'0.17+Ca'1.24 was the total flavonoids characteristically lower at the earlier 122 and 136 DAFB periods and significantly highest at $150 \mathrm{DAFB}$, showing a rather increasing trend as fruit maturity progressed.

\section{2. 'Golden Delicious' Apples}

A significant interaction ( $\left.\mathrm{B}+\mathrm{Ca}^{\star} \mathrm{DAFB}\right)$ effect was recorded for both 'Golden Delicious' fruit peel total phenolics and total flavonoids means (Table 8). At 80 DAFB, no treatment significantly differed with the control, and generally as well among the treatments, except B'0.02+Ca'0.06+Zn'0.03 (lower total phenolics) 
Table 8. 'Golden Delicious' apple peel phenolics.

\begin{tabular}{|c|c|c|c|c|c|}
\hline Phenolic & Treatments $(\mathrm{B}+\mathrm{Ca})$ & \multicolumn{4}{|c|}{ Days after full bloom (DAFB) } \\
\hline \multirow{6}{*}{$\mathrm{TP}^{1}$} & & 80 & 94 & 108 & 122 \\
\hline & $\mathrm{B}^{\prime} 0.00+\mathrm{Ca}^{\prime} 0.00$ & $249^{\text {bcde }}$ & $333^{\mathrm{a}}$ & $166^{\mathrm{fgh}}$ & $191^{\mathrm{efg}}$ \\
\hline & $\mathrm{B}^{\prime} 0.02+\mathrm{Ca}^{\prime} 1.24$ & $292^{\mathrm{abc}}$ & $243^{\text {bcde }}$ & $161^{\text {gh }}$ & $149^{\text {gh }}$ \\
\hline & $\mathrm{B}^{\prime} 0.02+\mathrm{Ca}^{\prime} 0.06+Z \mathrm{Zn}^{\prime} 0.03$ & $234^{\text {cdef }}$ & $265^{\mathrm{abcd}}$ & $148^{\mathrm{gh}}$ & $200^{\operatorname{defg}}$ \\
\hline & $\mathrm{B}^{\prime} 0.08+\mathrm{Ca}^{\prime} 1.24$ & $305^{\mathrm{ab}}$ & $264^{\mathrm{bcd}}$ & $245^{\text {bcde }}$ & $122^{\mathrm{h}}$ \\
\hline & $\mathrm{B}^{\prime} 0.17+\mathrm{Ca}^{\prime} 1.24$ & $269^{\mathrm{abcd}}$ & $268^{\mathrm{abcd}}$ & $209^{\operatorname{defg}}$ & $204^{\operatorname{defg}}$ \\
\hline & $\mathrm{B}^{\prime} 0.00+\mathrm{Ca}^{\prime} 0.00$ & $156^{\mathrm{bc}}$ & $206^{\mathrm{a}}$ & $130^{\text {cdef }}$ & $115^{\text {efgh }}$ \\
\hline & $\mathrm{B}^{\prime} 0.02+\mathrm{Ca}^{\prime} 1.24$ & $174^{\mathrm{b}}$ & $162^{\mathrm{b}}$ & $119^{\operatorname{defg}}$ & $98^{\mathrm{gh}}$ \\
\hline $\mathrm{TF}^{2}$ & $\mathrm{~B}^{\prime} 0.02+\mathrm{Ca}^{\prime} 0.06+\mathrm{Zn}^{\prime} 0.03$ & $146^{\text {bcde }}$ & $165^{\mathrm{b}}$ & $112^{\mathrm{fgh}}$ & $116^{\text {defg }}$ \\
\hline & $\mathrm{B}^{\prime} 0.08+\mathrm{Ca}^{\prime} 1.24$ & $167^{\mathrm{b}}$ & $162^{\mathrm{b}}$ & $168^{\mathrm{b}}$ & $84^{\mathrm{h}}$ \\
\hline & B'0.17+Ca'1.24 & $156^{\mathrm{bc}}$ & $172^{\mathrm{b}}$ & $147^{\mathrm{bcd}}$ & $123^{\text {defg }}$ \\
\hline
\end{tabular}

$\mathrm{TP}=$ Total Phenolics $\left({ }^{1} \mathrm{mg}\right.$ GAE. $100 \mathrm{~g}^{-1}$ fresh weight, $\left.\mathrm{p}\left(\mathrm{B}+\mathrm{Ca}{ }^{*} \mathrm{DAFB}\right)=0.0114\right)$ and $\mathrm{TF}=$ Total Flavonoids, $\left({ }^{2} \mathrm{mg}\right.$ CE. $100 \mathrm{~g}^{-1}$ fresh weight, $\left.p\left(\mathrm{~B}+\mathrm{Ca}^{*} \mathrm{DAFB}\right)=0.0047\right)$.

that differed significantly with B'0.08+Ca'1.24 (higher total phenolics). At 94 $\mathrm{DAFB}$, the control showed the highest means that were significantly different from all values of the treatments, except the total phenolics means of both treatments $B^{\prime} 0.02+\mathrm{Ca}^{\prime} 0.06+Z n^{\prime} 0.03$ and B'0.17+Ca'1.24. All treatments had insignificant differences in total phenolics and total flavonoids means at 94 DAFB. At 108 $\mathrm{DAFB}$, the control had indifferent mean as all treatments except with treatment B'0.08+Ca'1.24 which had the highest total phenolics that also did not differ with only the total phenolics level of treatment $\mathrm{B}^{\prime} 0.17+\mathrm{Ca}^{\prime} 1.24$. These two treatments B'0.08+Ca'1.24 and B'0.17+Ca'1.24 had the highest and indifferent total flavonoids means at $108 \mathrm{DAFB}$, but only treatment $\mathrm{B}^{\prime} 0.08+\mathrm{Ca}^{\prime} 1.24$ differed significantly from the control, whereas treatment $\mathrm{B}^{\prime} 0.17+\mathrm{Ca}^{\prime} 1.24$ was only significantly different from treatment $\mathrm{B}^{\prime} 0.02+\mathrm{Ca}^{\prime} 0.06+\mathrm{Zn}$ ' $^{\prime} .03$ which had lowest total flavonoids mean at that particular fruit maturity stage. At $122 \mathrm{DAFB}$, treatment B'0.08+Ca'1.24 with the lowest total phenolics mean was significantly different from all other treatments including the control, except B'0.02+Ca'1.24. However, the lowest total flavonoids of the same $\mathrm{B}^{\prime} 0.08+\mathrm{Ca}^{\prime} 1.24$ treatment was only significantly different from that of treatments B'0.02+Ca'0.06+Zn'0.03 and B'0.17+Ca'1.24.

With advance of DAFB, the control's highest total phenolics and total flavonoids means at 94 DAFB significantly differed from means at 108 and 122 $\mathrm{DAFB}$, generally showing a decreasing trend as 'Golden Delicious' fruit maturity progressed. The total phenolics and total flavonoids means associated with B'0.02+Ca'1.24 at both 80 and 94 DAFB were not different as well as means at both 108 and 122 DAFB, but these pairs significantly differed from each other, hence a significant decrease as DAFB advanced occurred. The total phenolics means of B'0.02+Ca'0.06+Zn'0.03 at 80, 94 and 122 DAFP periods did not differ as was also means at 108 and $122 \mathrm{DAFB}$, suggesting no definite increase or de- 
crease of trend was observed and as well for the total flavonoids means of this $\mathrm{Zn}$ treatment. The total phenolics and total flavonoids means for treatment B'0.08+Ca'1.24 did not show significant differences from 80 to 108 DAFB, but all these means differed with its lowest mean recorded at 122 DAFB, inferring a later sharp decrease towards harvest maturity in this treatment. For treatment B'0.17+Ca'1.24, total phenolics means were not significantly different across all DAFB periods, however, the total flavonoids means showed insignificant differences from 80 to 108 DAFB, and its lowest total flavonoids mean at 122 DAFB was only not different from the value at 108 DAFB.

The total flavonoids to total phenolics ratio means had insignificant treatment as well as interaction (B+Ca*DAFB) effects (Table 9). The significant DAFB effect showed that total flavonoids to total phenolics ratio means recorded at 80 , 94 and 122 DAFB were the lowest overall indicating increases as 80 to 108 DAFB, and sharp decrease by 122 DAFB. In addition, there were insignificant differences among treatments for the studied physico-chemical attributes in 'Golden Delicious' fruit at harvest (Table 10). Total soluble solids content of sun exposed fruit side (SE) was higher compared to unexposed fruit side (NSE). Respective standard errors (SE) associated with means of the sun exposed fruit side (SE) and unexposed fruit side (NSE) showed that only total soluble solids SE with treatments B'0.02+Ca'0.06+Zn'0.03, B'0.02+Ca'1.24 and B'0.17+Ca'1.24 was significantly different from its corresponding total soluble solids NSE.

Table 9. 'Golden Delicious' apple peel total flavonoids to phenolics ratio (TF:TP).

\begin{tabular}{|c|c|}
\hline Source & TF:TP (\%) \\
\hline \multicolumn{2}{|l|}{ Treatments $(\mathrm{B}+\mathrm{Ca})$} \\
\hline $\mathrm{B}^{\prime} 0.00+\mathrm{Ca}^{\prime} 0.00$ & $66.60^{\mathrm{ns}}$ \\
\hline B'0.02+Ca'1.24 & 69.15 \\
\hline $\mathrm{B}^{\prime} 0.02+\mathrm{Ca}^{\prime} 0.06+\mathrm{Zn} 0.03$ & 65.80 \\
\hline B'0.08+Ca'1.24 & 66.95 \\
\hline B'0.17+Ca'1.24 & 64.90 \\
\hline$p$ & 0.8825 \\
\hline \multicolumn{2}{|l|}{ Days after full bloom (DAFB) } \\
\hline 80 & $60.48^{\mathrm{b}}$ \\
\hline 94 & $64.28^{\mathrm{b}}$ \\
\hline 108 & $76.44^{\mathrm{a}}$ \\
\hline 122 & $65.52^{\mathrm{b}}$ \\
\hline$p$ & 0.0004 \\
\hline$p\left(\mathrm{~B}+\mathrm{Ca}^{*} \mathrm{DAFB}\right)$ & 0.4126 \\
\hline
\end{tabular}


Table 10. 'Golden Delicious' apple fruit physico-chemical attributes at harvest.

\begin{tabular}{ccccc}
\hline Treatments (B+Ca) & \multicolumn{4}{c}{ Physico-chemical attributes } \\
\hline & FD & FM & TSS-SE & TSS-NSE \\
\cline { 2 - 5 } B'0.00+Ca'0.00 & $70.63^{\text {ns }}$ & $155.00^{\text {ns }}$ & $15.37 \pm 0.43$ & $14.68 \pm 0.37$ \\
B'0.02+Ca'1.24 & 68.63 & 147.67 & $15.68 \pm 0.39$ & $15.18 \pm 0.57$ \\
B'0.02+Ca'0.06+Zn'0.03 & 71.96 & 163.83 & $15.41 \pm 0.49^{*}$ & $14.18 \pm 0.45^{*}$ \\
B'0.08+Ca'1.24 & 69.29 & 148.33 & $16.08 \pm 0.35^{*}$ & $14.69 \pm 0.09^{*}$ \\
B'0.17+Ca'1.24 & 69.67 & 152.20 & $15.23 \pm 0.65^{*}$ & $14.16 \pm 0.40^{*}$ \\
$p$ & 0.6530 & 0.7500 & 0.7360 & 0.5420 \\
\hline
\end{tabular}

$\mathrm{FD}=$ Fruit Diameter $(\mathrm{mm}), \mathrm{FM}=$ Fruit Mass $(\mathrm{g}), \mathrm{TSS}=$ Total soluble solids $(\%$ Brix), $\mathrm{SE}=$ Sun Exposed side of fruit, NSE = Unexposed side of fruit and ${ }^{*}$ significant differences between corresponding SE and NSE data.

\section{3. 'Granny Smith' Apples}

There was a significant interaction $\left(B+C a^{*} \mathrm{DAFB}\right)$ effect on the 'Granny Smith' fruit peel total phenolics and total flavonoids means (Table 11). At 94 DAFB, differences were not significant for total phenolics and total flavonoids means of control, $\mathrm{B}^{\prime} 0.02+\mathrm{Ca}^{\prime} 0.06+\mathrm{Zn}^{\prime} 0.03$ and $\mathrm{B}^{\prime} 0.08+\mathrm{Ca}^{\prime} 1.24$, as well as between $\mathrm{B}^{\prime} 0.02+\mathrm{Ca}^{\prime} 0.06+\mathrm{Zn} \mathrm{n}^{\prime} 0.03$ and $\mathrm{B}^{\prime} 0.17+\mathrm{Ca}^{\prime} 1.24$, and $\mathrm{B}^{\prime} 0.02+\mathrm{Ca}^{\prime} 1.24$ and $\mathrm{B}^{\prime} 0.17+\mathrm{Ca}{ }^{\prime} 1.24$. At $108 \mathrm{DAFB}$, total phenolics and total flavonoids mean differences of control and $\mathrm{B}^{\prime} 0.02+\mathrm{Ca}^{\prime} 1.24$, control and $\mathrm{B}^{\prime} 0.08+\mathrm{Ca}^{\prime} 1.24$ were not significant. In addition, the total phenolics and total flavonoids mean differences among treatments $\mathrm{B}^{\prime} 0.02+\mathrm{Ca}^{\prime} 1.24, \mathrm{~B}^{\prime} 0.02+\mathrm{Ca}^{\prime} 0.06+Z n^{\prime} 0.03$ and $\mathrm{B}^{\prime} 0.17+\mathrm{Ca}^{\prime} 1.24$ were not significant. At $122 \mathrm{DAFB}$, total phenolics and total flavonoids means of the control (no $\mathrm{B}+\mathrm{Ca}$ ) did not differ significantly with all treatments, except B'0.08+Ca'1.24. The total phenolics and total flavonoids means in all $\mathrm{B}+\mathrm{Ca}$ treatments did not differ significantly. At $136 \mathrm{DAFB}$, no significant differences in total phenolics and total flavonoids between control and treatments occurred, and only treatments $\mathrm{B}^{\prime} 0.02+\mathrm{Ca}^{\prime} 0.06+\mathrm{Zn}^{\prime} 0.03$ (low total phenolics and total flavonoids) and B'0.17+Ca'1.24 (high total phenolics and total flavonoids) differed significantly.

In addition, fruit maturity progress from 108 to $136 \mathrm{DAFB}$, the total phenolics and total flavonoids means in control, B'0.02+Ca'1.24, B'0.02+Ca'0.06+Zn'0.03 and $\mathrm{B}^{\prime} 0.17+\mathrm{Ca}^{\prime} 1.24$ did not differ significantly (Table 11 ), showing that generally as fruit maturity progressed, the total phenolics and total flavonoids means did not significantly decrease or increase. However, only treatment B'0.08+Ca'1.24 yielded significantly different total phenolics and total flavonoids at 94 and 108 DAFB in comparison to both at 122 and $136 \mathrm{DAFB}$, showing a significant decline as fruit maturity advanced. Means of total flavonoids to total phenolics ratio did not have significant treatment and interaction $\left(\mathrm{B}+\mathrm{Ca}^{*} \mathrm{DAFB}\right)$ (Table 12), only the DAFB periods had a significant impact. Generally, total flavonoids to total phenolics ratio means showed decreasing values from 94 to 136 DAFB, but only significant dif- 
ferences occurred between 94 DAFB and both 122 and 136 DAFB values.

The physico-chemical attributes assessed at fruit harvest had insignificant mean differences (Table 13). The total soluble solids of sun exposed fruit side (SE) were higher compared to unexposed fruit side (NSE). Respective standard errors associated with means of SE and NSE data showed that fruit under B'0.08+Ca'1.24 had insignificant differences in total soluble solids between the SE and NSE.

Table 11. 'Granny Smith' apple peel phenolics.

\begin{tabular}{|c|c|c|c|c|c|}
\hline \multirow[t]{2}{*}{ Phenolic } & \multirow[t]{2}{*}{ Treatments $(\mathrm{B}+\mathrm{Ca})$} & \multicolumn{4}{|c|}{ Days after full bloom (DAFB) } \\
\hline & & 94 & 108 & 122 & 136 \\
\hline \multirow{5}{*}{$\mathrm{TP}^{1}$} & $\mathrm{~B}^{\prime} 0.00+\mathrm{Ca}^{\prime} 0.00$ & $523^{\mathrm{a}}$ & $431^{\mathrm{abcd}}$ & $441^{\mathrm{abcd}}$ & $122^{\text {cdef }}$ \\
\hline & B'0.02+Ca'1.24 & $320^{\text {def }}$ & $337^{\text {cdef }}$ & $327^{\text {cdef }}$ & $309^{\text {def }}$ \\
\hline & $\mathrm{B}^{\prime} 0.02+\mathrm{Ca}^{\prime} 0.06+\mathrm{Zn} 0.03$ & $458^{\mathrm{abc}}$ & $261^{\mathrm{ef}}$ & $331^{\text {cdef }}$ & $229^{f}$ \\
\hline & B'0.08+Ca'1.24 & $503^{\mathrm{ab}}$ & $483^{\mathrm{ab}}$ & $278^{\mathrm{ef}}$ & $272^{\text {ef }}$ \\
\hline & B'0.17+Ca'1.24 & $346^{\text {cdef }}$ & $284^{\mathrm{ef}}$ & $315^{\text {def }}$ & $371^{\text {bcde }}$ \\
\hline \multirow{5}{*}{$\mathrm{TF}^{2}$} & $\mathrm{~B}^{\prime} 0.00+\mathrm{Ca}^{\prime} 0.00$ & $279^{\mathrm{a}}$ & $225^{\mathrm{abcd}}$ & $227^{\mathrm{abcd}}$ & $173^{\text {def }}$ \\
\hline & B'0.02+Ca'1.24 & $185^{\text {cdef }}$ & $182^{\text {def }}$ & $172^{\text {def }}$ & $148^{\mathrm{ef}}$ \\
\hline & B'0.02+Ca'0.06+Zn'0.03 & $257^{\mathrm{ab}}$ & $147^{\mathrm{ef}}$ & $170^{\text {def }}$ & $123^{\mathrm{f}}$ \\
\hline & B'0.08+Ca'1.24 & $272^{\mathrm{a}}$ & $244^{\mathrm{abc}}$ & $146 \mathrm{ef}$ & $146^{\mathrm{ef}}$ \\
\hline & B’0.17+Ca'1.24 & $202^{\text {bcde }}$ & $154^{\mathrm{ef}}$ & $170^{\mathrm{def}}$ & $188^{\text {cde }}$ \\
\hline
\end{tabular}

$\mathrm{TP}=$ Total Phenolics $\left({ }^{1} \mathrm{mg}\right.$ GAE. $100 \mathrm{~g}^{-1}$ peel fresh weight, $\left.p\left(\mathrm{~B}+\mathrm{Ca}{ }^{*} \mathrm{DAFB}\right)=0.0227\right)$, TF $=$ Total Flavonoids, $\left({ }^{2} \mathrm{mg} \mathrm{CE} .100 \mathrm{~g}^{-1}\right.$ peel fresh weight, $\left.p\left(\mathrm{~B}+\mathrm{Ca}^{*} \mathrm{DAFB}\right)=0.0230\right)$.

Table 12. 'Granny Smith' apple peel total flavonoids to phenolics ratio (TF:TP).

\begin{tabular}{cc}
\hline Source & TF:TP (\%) \\
\hline Treatments $(\mathrm{B}+\mathrm{Ca})$ & $52.16^{\mathrm{ns}}$ \\
B'$^{\prime} 0.00+\mathrm{Ca}^{\prime} 0.00$ & 53.56 \\
B'$^{\prime} 0.02+\mathrm{Ca}^{\prime} 1.24$ & 54.58 \\
B'0.02+Ca'0.06+Zn'0.03 & 55.15 \\
B'0.08+Ca'1.24 & 53.34 \\
B'$^{\prime} 0.17+\mathrm{Ca}^{\prime} 1.24$ & 0.3700 \\
$p$ & \\
Days after full bloom (DAFB) & $56.50^{\mathrm{a}}$ \\
94 & $54.00^{\mathrm{ab}}$ \\
108 & $53.02^{\mathrm{b}}$ \\
122 & $51.51^{\mathrm{b}}$ \\
136 & 0.0061 \\
$p$ & 0.3583 \\
$p\left(\mathrm{~B}+\mathrm{Ca}{ }^{*} \mathrm{DAFB}\right)$ &
\end{tabular}


Table 13. 'Granny Smith' apple fruit physico-chemical attributes at harvest.

\begin{tabular}{ccccc}
\hline Treatments (B+Ca) & \multicolumn{4}{c}{ Physico-chemical attributes } \\
\hline & FD & FM & TSS-SE & TSS-NSE \\
\cline { 2 - 5 } B'0.00+Ca'0.00 & $70.21^{\text {ns }}$ & $152.80^{\text {ns }}$ & $14.25 \pm 0.23^{*}$ & $13.13 \pm 0.27^{*}$ \\
B'0.02+Ca'1.24 & 69.42 & 149.17 & $14.11 \pm 0.38^{*}$ & $13.25 \pm 0.49^{*}$ \\
B'0.02+Ca'0.06+Zn'0.03 & 67.54 & 136.33 & $14.71 \pm 0.63^{*}$ & $13.09 \pm 0.33^{*}$ \\
B'0.08+Ca'1.24 & 73.59 & 178.30 & $13.85 \pm 0.10$ & $13.75 \pm 0.40$ \\
B'0.17+Ca'1.24 & 72.67 & 171.25 & $13.61 \pm 0.22^{*}$ & $12.58 \pm 0.16^{*}$ \\
$p$ & 0.1930 & 0.1850 & 0.3040 & 0.2700 \\
\hline
\end{tabular}

$\mathrm{FD}=$ Fruit Diameter $(\mathrm{mm}), \mathrm{FM}=$ Fruit Mass $(\mathrm{g}), \mathrm{TSS}=$ Total soluble solids $(\%$ Brix $), \mathrm{SE}=$ Sun Exposed side of fruit, NSE = Unexposed side of fruit, and *significant mean differences between corresponding SE and NSE data.

\section{Discussion}

\subsection{Rationale of the Study/Experimental Approach}

The 2014/15 'Cripps Pink' experiment served two purposes; 1) explore influence of fruit position and orientation, and importance of anchoring such studies at early fruit maturity stages, and 2) assess importance varying $\mathrm{Ca}$ concentration using treatments $\mathrm{B}^{\prime} 0.02+\mathrm{Ca}^{\prime} 0.10$ and $\mathrm{B}^{\prime} 0.02+\mathrm{Ca}^{\prime} 1.29$ versus control $\left(\mathrm{B}^{\prime} 0.00+\mathrm{Ca}^{\prime} 0.00\right)$. With early fruit maturity stages (Table 2 ), no significant treatment or interaction $\left(B+C a^{\star} D A F B\right)$ effects were recorded and this could not enable an effective assessment of treatment effect on peel phenolics at this early fruit maturity stage. As thus, further fruit sampling was conducted at optimum harvest (184 DAFB), and also the 2014/15 experiment was deliberately harvested late (214 DAFB) with intention to increase contrasts between treatments.

Findings in Table 2 possibly indicated that treatment effects are not realized at young fruit stages. This supposition is supported by results in Table 3 and Table 4 where, 1) total flavonoids to total phenolics ratio showed significant interaction $\left(\mathrm{B}+\mathrm{Ca}^{*} \mathrm{DAFB}\right)$ effects at $184 \mathrm{DAFB}$, and 2) foliar $\mathrm{B}+\mathrm{Ca}$ treatments showed evidence of significant total flavonoids to total phenolics ratio mean differences between the West and East oriented fruit. Therefore, focus in 2015/16 experiments shifted to fruit maturity stages towards optimum harvest maturity and in outer canopy fruit and both sides of the trees as also results of 214 DAFB did not have any case of significant interaction effect (Table 5).

This study utilized three different cultivars that also have varying fruiting characteristics and harvest maturity periods. It is expected that peel biochemical profile will vary with such cultivar differences [21], therefore cultivars were considered in statistical analyses. Cultivars used in this study were also on similar rootstocks (M793), in order to eliminate the possibility of different rootstock effects which reportedly impart effects on tree and fruit physiology as well [22]. In addition, the inclusion of $\mathrm{Zn}$ treatment in 2015/16 was because $\mathrm{Zn}$ is also reportedly important in plant abiotic stress tolerance and recommended for foliar application in apple orchards [23]. 


\section{2. 'Cripps Pink' Peel Phenolics}

The bulk of phenolic synthesis in apple fruit occurs between 30 and 60 DAFB where after the rapidly increasing fruit size leads to a reduction in phenolic concentrations by means of 'dilution' [24]. This explains the observed significant increase in 'Cripps Pink' peel phenolics from 35 to 56 DAFB, and the subsequent decrease by 70 DAFB (Table 2). The significant total flavonoids mean differences between East and West oriented fruit (Table 3) is likely because East fruit is more exposed to the sun than West in this particular orchard [3]. In 2015/16, 'Cripps Pink' apple peel total phenolics showed significant decreases as fruit matured (Table 6), possibly due to increasing fruit size and associated "dilution" effect [24] [25] Lister. However, total flavonoids to total phenolics ratio means showed significant increases from 122 to $150 \mathrm{DAFB}$, possibly an attribute of the increasing flavonoid-anthocyanins as 'Cripps Pink' is a bi-color fruiting cultivar whose fruit is green when young and blushes to red with advanced maturity [25]. From Table 6 and Table 7, total flavonoids means peaked at 150 DAFB, and at this maturity stage, 'Cripps Pink' apple fruit is expected to attain the second and last peak of the peel anthocyanin accumulation [26]. Therefore, this translates into a large increase in total flavonoids against total phenolics levels explaining the observed significant rise of total flavonoids to total phenolics ratio means with DAFB.

The significant interaction $\left(\mathrm{B}+\mathrm{Ca}{ }^{\star} \mathrm{DAFB}\right)$ effect on total flavonoids (Table 7) forms a firm indication that foliar $\mathrm{B}+\mathrm{Ca}$ has an effect on 'Cripps Pink' apple peel phenolic aspects as fruit maturity progresses (advancing DAFB). The important aspects of this significant interaction are, 1) significant differences between total flavonoids means of $\mathrm{B}^{\prime} 0.08+\mathrm{Ca}^{\prime} 1.24$ and $\mathrm{B}^{\prime} 0.17+\mathrm{Ca}^{\prime} 1.24$ at $122 \mathrm{DAFB}$ and $\mathrm{B}^{\prime} 0.02+\mathrm{Ca}^{\prime} 1.24$ and $\mathrm{B}^{\prime} 0.17+\mathrm{Ca}^{\prime} 1.24$ at $136 \mathrm{DAFB}$ as well as 2) significant differences between total flavonoids at $136 \mathrm{DAFB}$ (low) and 136 to $150 \mathrm{DAFB}$ periods with $B^{\prime} 0.02+C^{\prime} 0.06+Z n^{\prime} 0.03$. In addition, 3) significantly lower total flavonoids at 164 DAFB from all earlier DAFB periods with treatment B'0.08+Ca'1.24. Notably, these hallmarks of the significant interaction (B+Ca*DAFB) for 'Cripps Pink' peel total flavonoids levels do not yield any meaningful interpretation to attribute to the varying B or Ca levels in treatments or even as an effect of inclusion of $\mathrm{Zn}$ because in all cases 1 ) and 2), the treatments did not significantly differ from the control (no $\mathrm{B}+\mathrm{Ca}$ ). In addition, at no DAFB period did the total flavonoids means of the $\mathrm{Zn}$ treatment significantly differ from any treatment and control.

Generally from Table 7, no treatment could adequately show a clear pattern to classify as increased or decreased total flavonoids result, as all treatments were not significantly different from the control or from each other across the DAFB periods. The 2014/15 data (Table 3), had resulted in total flavonoids means being under significant interaction $\left(\mathrm{B}+\mathrm{Ca}{ }^{*} \mathrm{O}\right)$ effect and together with results in Table 7, evidence that foliar $\mathrm{B}+\mathrm{Ca}$ may be influencing dynamics of 'Cripps Pink' peel flavonoids as fruit maturity progresses is confirmed. However, the lack of 
the desired (as for this study) clearly distinguishing patterns in the interaction $\left(\mathrm{B}+\mathrm{Ca}^{\star} \mathrm{DAFB}\right)$ effect analysis as presented in Table 7 , may infer that the foliar $\mathrm{B}+\mathrm{Ca}$ effect on 'Cripps Pink' peel flavonoids may be revealed by using more robust methods that target the levels of specific flavonoids types as fruit maturity progresses.

\section{3. 'Golden Delicious' Peel Phenolics and Physico-Chemical Attributes}

The significant interaction $\left(\mathrm{B}+\mathrm{Ca}^{\star} \mathrm{DAFB}\right)$ effect (Table 8) indicates the influence of foliar $\mathrm{B}+\mathrm{Ca}$ on phenolic levels as the GA progressed with maturity (advancing DAFB). However, as was observed with the 'Cripps Pink' apple peel total flavonoids means $\mathrm{B}+\mathrm{Ca}^{*} \mathrm{DAFB}$ effect analysis (Table 7 ), there was no treatment that yielded clear distinguished increase or decrease of total phenolics or total flavonoids means as DAFB progressed. All treatment total phenolics and total flavonoids levels were not significantly different amongst themselves or from the control at $80 \mathrm{DAFB}$, and only B'0.08+Ca'1.24 (higher total phenolics) and B'0.02+Ca'0.06+Zn'0.03 (lower total phenolics) had significant differences, but this cannot be attributed to treatment mineral differences as they both did not significantly differ from the control or other treatments. The same analogy applies to respective significant treatment differences in total phenolics and total flavonoids means at 94, 108 and 122 DAFB. At these fruit maturity stage, the high B $\left(0.17 \mathrm{~g}^{-1} / 0.08 \mathrm{~g}^{-1}\right)$, low B $\left(0.02 \mathrm{~g}^{-1}\right)$ and $\mathrm{Zn}$ with low $\mathrm{Ca}\left(0.06 \mathrm{~g}^{-1}\right)$ containing treatments had several cases of insignificant differences amongst themselves as well as with the control (no $\mathrm{B}+\mathrm{Ca}$ ).

Foliar $\mathrm{B}+\mathrm{Ca}$ treatment effect on total flavonoids to total phenolics ratio means was not tangible ( $\mathrm{p}$-value 0.8825) (Table 8). But, DAFB periods had a significant effect on total flavonoids to total phenolics ratio means ( $p$-value 0.0004 ), and rose with advancing DAFB peaking at 108 DAFB (Table 9), comparable to the total flavonoids to total phenolics ratio trend in 'Cripps Pink' (Table 6). But, contrary to 'Cripps Pink', the total flavonoids means and the total flavonoids to total phenolics ratio means peak in 'Golden Delicious' at 108 DAFB did not clearly match, this should be attributed to mere cultivar differences. However, green fruiting apples have also been reported to show an increment of flavonoids and general reduction in phenolic levels as fruit maturity advances [27] [28] [29] [30] [31] [32], and this phenomenon should also be responsible for the observed total flavonoids to total phenolics ratio rise from 80 to 108 in 'Golden Delicious' apples.

There were no significant differences among foliar $\mathrm{B}+\mathrm{Ca}$ treatments for the studied physico-chemical attributes in 'Golden Delicious' apple fruit at harvest (Table 12). Apple fruit sides exposed to the sun have higher total soluble solids [33]. Generally, total soluble solids of sun exposed fruit side (SE) were higher compared to the unexposed fruit side (NSE). However, significant differences in total soluble solids among SE and NSE data for treat- 
ments B'0.02+Ca'0.06+Zn'0.03, B'0.02+Ca'1.24 and B'0.17+Ca'1.24, further demonstrates that varying $\mathrm{B}, \mathrm{Ca}$ or inclusion of $\mathrm{Zn}$ did not have a profound effect.

\section{4. 'Granny Smith' Peel Phenolics and Physico-Chemical Attributes}

The significant interaction (B+Ca*DAFB) for 'Granny Smith' apple peel total phenolics and total flavonoids means (Table 11) shows the importance of the foliar $\mathrm{B}+\mathrm{Ca}$ effect alongside DAFB periods. However, the effect of varying the $\mathrm{B}$, Ca or inclusion of Zn on the 'Granny Smith' total phenolics and total flavonoids means as DAFB advanced is not well defined by the results obtained. This is because as was observed with 'Cripps Pink' 2015/16 apple peel total flavonoids means (Table 7), 'Golden Delicious' apple peel total phenolics and total flavonoids means (Table 8), there is no clear trends to be able to resolve effects of the foliar $\mathrm{B}+\mathrm{Ca}$ treatments. For instance, at $94 \mathrm{DAFB}$, the highest $\mathrm{B}$ treatment (B'0.17+Ca'1.24) had insignificant total phenolics and total flavonoids differences with B'0.02+Ca'1.24 which had low B levels, and the low B, Ca plus Zn (B’0.02+Ca'0.06+Zn'0.03), moderate B and high Ca (B'0.08+Ca'1.24) were not significantly different from the control (no $\mathrm{B}+\mathrm{Ca}$ ) for both total phenolics and total flavonoids at $94 \mathrm{DAFB}$. This scenario of varying high and low B plus Ca and $\mathrm{Zn}$ inclusion treatment (s) not differing amongst themselves as well as with the control is evident at almost all the DAFB periods in Table 11.

The total flavonoids to total phenolics ratio levels in 'Granny Smith' had insignificant treatment as well as interaction $\left(B+\mathrm{Ca}^{\star} \mathrm{DAFB}\right)$ effect, and the significant DAFB effect showed decreasing trend with advance of DAFB as values recorded at 94 (high) and 136 DAFB (low) were significantly different, contrary to what was observed in both 'Cripps Pink' (Table 6) and 'Golden Delicious' (Table 9) total flavonoids to total phenolics ratio data, providing another instance of cultivar differences in the context of this study, as 'Cripps Pink' and 'Golden Delicious' are genetically related [25]. The 'Granny Smith' apple peel total flavonoids to total phenolics ratio levels were rather similar at most DAFB periods as differences were not significant between 94 and 108 DAFB as well as between 108, 122 and 136 DAFB periods. This does not therefore provide an opposing ground to previous findings on apple peel phenolics reduction changes with advancing fruit development.

No significant differences among treatments for the studied physico-chemical attributes were recorded in 'Granny Smith' fruit at harvest. Total soluble solids of sun exposed fruit side (SE) was higher compared to unexposed fruit side (NSE) in agreement with previous studies [Hamadziripi, 2012], as was the case in 'Golden Delicious' fruit as well (Table 10). Only treatment B'0.08+Ca'1.24 displayed insignificant total soluble solids differences between the SE and NSE data, and this as well shows that total soluble solids was independent of the varying $B$ and Ca levels as well as inclusion of $\mathrm{Zn}$ in the treatments as was the case with 'Golden Delicious' fruit. 


\subsection{Significant Peel Phenolic B Plus Ca Effect Is Likely on Flavonoids}

Cultivar differences and fruit maturity stages (DAFB periods) are appearing more important in phenolic dynamics than foliar $\mathrm{B}+\mathrm{Ca}$ treatment differences in all cultivars. In all cultivars, the DAFB factor significantly influenced the phenolic parameters (Table 2, Table 6, Table 9 and Table 12), where particularly a decreasing total phenolics or relatively increasing total flavonoids to total phenolics ratio trend occurred. The decreasing phenolic attribute of apple fruit with advance of DAFB (fruit maturity) peels is well reported phenomenon in several previous studies [28] [29] [30] [31] [32]. Foliar B+Ca treatments did not have significant effect on phenolic parameters measures including where significant interaction $\left(\mathrm{B}+\mathrm{Ca}^{\star} \mathrm{DAFB}\right)$ effects occurred except for 'Granny Smith' apple peel total phenolics and total flavonoids means, for instance, $\mathrm{B}+\mathrm{Ca}$ effect $\mathrm{p}$-value was 0.0830, 0.3449, 0.1825 respectively for 'Cripps Pink' 2015/16 total flavonoids and 'Golden Delicious' apple peel total phenolics and total flavonoids data. The foliar $\mathrm{B}+\mathrm{Ca}$ effect p-value on 'Granny Smith' apple peel total phenolics and total flavonoids means was 0.0025 . This observation raises the possibility that the effect of foliar $\mathrm{B}+\mathrm{Ca}$ could be significant with specific flavonoid types in the apple peel, and the significant $\mathrm{B}+\mathrm{Ca}$ effect on the green fruiting and maturing 'Granny Smith' could be having more flavonoids in its peel phenolic composition compared to both 'Cripps Pink' and 'Golden Delicious' apples.

The lack of a clear definable foliar B+Ca effect on influencing the apple peel phenolic means in clear patterns amidst cultivar differences could be due to; 1) the inevitable "mild" nature of foliar $\mathrm{B}+\mathrm{Ca}$ treatments since boron toxicity can occur easily in plants [34] [35], and 2) the generic-ness of the techniques applied in this study to quantify apple peel total phenolics and total flavonoids levels. Although Gallic acid (GAE) and Catechin (CE) equivalents are widely used methods to quantify these phenolic parameters in apple peels, several types/forms of these phenolics exist [36] [37] [38]. Therefore, as earlier mentioned, further investigations should focus on major peel flavonoid types or alternatively flavanone 3-hydroxylase (F3H, EC 1.4.11.9). The enzyme flavanone 3-hydroxylase is heavily involved in the biosynthesis of flavonoids and has important roles in resistance to abiotic stress including apple fruit [16] [39] [40] [41]. Further still, the roles of this enzyme arouse interest in peel biochemicals that are relevant within the context of this study, for instance chlorophyll pigments under such varying foliar $\mathrm{B}+\mathrm{Ca}$ treatments. Chlorophyll and associated photosynthetic pigments may be under the protection of flavonoids [42] [43].

Physico-chemical attributes were not affected by the foliar $\mathrm{B}+\mathrm{Ca}$ treatments and this together with starch breakdown, fruit firmness among other fruit quality indicators assessed by [3], provide further support to assertions by [3] that quality of apple fruit was not compromised by the foliar B+Ca treatments. In addition, the behavior of total soluble solids from SE and NSS marching with established patterns is an indicator that none destructive methods especially 
Near Infra-Red Spectroscopy can be used to evaluate apple peel biochemistry under foliar $\mathrm{B}+\mathrm{Ca}$ treatments. In relation to this, and the proposition mentioned earlier to focus on flavanols in future studies, [44] used none destructive assessment of flavanols in apple fruit.

\section{Conclusion}

Cultivar differences and advance of days after full bloom were largely responsible for the results obtained for the three apple peel phenolic parameters. Therefore, the biochemical importance of varying boron, calcium and inclusion of zinc on dynamics of apple peel phenolics could not be precisely determined in this study. However, evidence of significant interaction effects for the apple peel total flavonoids in all cultivars demonstrated that the foliar boron plus calcium treatments had an effect on phenolic dynamics, and results from this study ably suggest future research in this area of study should focus on apple peel flavonoids and/or their major biosynthesis enzyme flavanone 3-hydroxylase. The focus on flavonoids pathway in apple fruit peels treated with foliar boron plus calcium formulations already known to suppress sunburn-browning incidence in apple orchards may yield clear treatment effects and hence better information to use in construing the sought underlying mode-of-action. In addition, flavonoids with roles of abiotic stress resistance in plant tissues may impact on availability and/or sustenance of other apple peel biochemicals that are affected during the abiotic stressful development of apple fruit sunburn phenomenon. As thus, apple peel pigments like chlorophylls should also be evaluated in apple fruit treated with foliar boron plus calcium treatment formulations in order to understand the other dimensions of the physiological impact on the apple peel.

\section{Acknowledgements}

Support for this research was obtained from Nulandis (Pty), South Africa and also through a capacity building competitive grant (RU/2015/DRRG/01/004) provided by Regional Universities Forum for Capacity Building in Agriculture (RUFORUM) to Makerere and Stellenbosch Universities.

\section{Conflicts of Interest}

The authors declare no conflicts of interest regarding the publication of this paper.

\section{References}

[1] Lötze, E. and Hoffman, E.W. (2014) Foliar Application of Calcium plus Boron Reduces the Incidence of Sunburn in 'Golden Delicious' Apple. Journal of Horticultural Science and Biotechnology, 89, 607-612. https://doi.org/10.1080/14620316.2014.11513127

[2] Lötze, E., Daiber, S.H. and Midgley, S.J.E. (2017) Boron in Combination with Calcium Reduces Sunburn in Apple Fruit. Boron, 2, 123-127.

[3] Daiber, S.H. (2017) Quantifying Changes in Tree Physiology after Amelioration to 
Reduce Sunburn on Apples. MSc Thesis, Stellenbosch University, Stellenbosch.

[4] Lötze, E., Daiber, S.H. and Midgley, S.J.E. (2018) Evaluating the Efficancy of a Preharvest Combination of Calcium and Boron as Foliar Application to Reduce Sunburn on 'Cripps Pink’ Apples. Acta Horticulture, 1217, 61-68. https://doi.org/10.17660/ActaHortic.2018.1217.7

[5] Mwije, A. (2017) Pioneering a New Paradigm of Sunburn Mitigation in Apples. In: Withers, J., Ed., Bringing Science to Communities: Voices from the Field, Issue 1, Ruforum Publication, Kampala, 20-23.

[6] Mwije, A. (2020) A Study of the Dynamics of Sunburn Reduction in Apple (Malus domestica) Using Foliar Applications of a Combination of Boron and Calcium. PhD Thesis, Stellenbosch University, Stellenbosch.

[7] Cakmak, I. and Romheld, V. (1997) Boron Deficiency-Induced Impairments of Cellular Functions in Plants. Plant and Soil, 193, 71-83. https://doi.org/10.1023/A:1004259808322

[8] Ruiz, J.M., Bretones, G., Baghour, M., Ragala, L., Belakbir, A. and Romero, L. (1998) Relationship between Boron and Phenolic Metabolism in Tobacco Leaves. Phytochemistry, 48, 269-272. https://doi.org/10.1016/S0031-9422(97)01132-1

[9] Hajiboland, R. and Farhanghi, F. (2010) Remobilization of Boron, Photosynthesis, Phenolic Metabolism and Anti-Oxidant Defense Capacity in Boron-Deficient Turnip (Brassica rapa L.) Plants. Soil Science and Plant Nutrition, 56, 427-437. https://doi.org/10.1111/j.1747-0765.2010.00478.x

[10] Hajiboland, R., Bahrami-Rad, S. and Bastani, S. (2013) Phenolics Metabolism in Boron-Deficient Tea [Camellia sinensis (L.) O. Kuntze] Plants. Acta Biologica Hungarica, 64, 196-206. https://doi.org/10.1556/ABiol.64.2013.2.6

[11] Conde, A., Pimentel, D., Neves, A., Dinis, L.T., Bernardo, S., Correia, C.M., Geros, H. and Moutinho-Pereira, J. (2016) Kaolin Foliar Application Has a Stimulatory Effect on Phenylpropanoid and Flavonoid Pathways in Grape Berries. Frontiers in Plant Science, 7, 1150. https://doi.org/10.3389/fpls.2016.01150

[12] Awad, M.A. and De Jager, A. (2002) Relationships between Fruit Nnutrients and Concentrations of Flavonoids and Chlorogenic Acid in 'Elstar' Apple Skin. Scientia Horticulturae, 92, 265-276. https://doi.org/10.1016/S0304-4238(01)00290-4

[13] Bizjak, J., Weber, N., Mikulic-Petkovsek, M., Slatnar, A., Stampar, F., Alam, Z., Stich, K., Halbwirth, H. and Veberic, R. (2013) Influence of Phostrade Ca on Color Development and Anthocyanin Content of 'Braeburn' Apple (Malus domestica Borkh.). HortScience, 48, 193-199. https://doi.org/10.21273/HORTSCI.48.2.193

[14] Yuri, J.A., Neira, A., Quilodran, A., Razmilic, I., Motomura, Y., Torres, C. and Palomo, I. (2010) Sunburn on Apples Is Associated with Increases in Phenolic Compounds and Antioxidant Activity as a Function of the Cultivar and Areas of the Fruit. Journal of Food, Agriculture and Environment, 8, 920-925.

[15] Racskó, J. and Schrader, L.E. (2012) Sunburn of Apple Fruit: Historical Background, Recent Advances and Future Perspectives. Critical Reviews in Plant Sciences, 31, 455-504. https://doi.org/10.1080/07352689.2012.696453

[16] Solovchenko, A. and Schmitz-Eiberger, M. (2003) Significance of Skin Flavonoids for UV-B-Protection in Apple Fruits. Journal of Experimental Botany, 54, 1977-1984. https://doi.org/10.1093/jxb/erg199

[17] Harb, J., Saleh, O., Kittemann, D., Neuwald, D.A., Frank, W. and Reski, R. (2013) Upregulation of Polyphenol-Related Genes Prevents 'skin burning' of Well-Colored 'Cameo' Apples Stored under Stressful Controlled Atmosphere Conditions. Postharvest Biology and Technology, 77, 121-127. 
https://doi.org/10.1016/j.postharvbio.2012.10.008

[18] Slinkard, K. and Singleton, V.L. (1977) Total Phenol Analysis: Automation and Comparison with Manual Methods. American Journal of Enology and Viticulture, 28, 49-55.

[19] Wolfe, K., Wu, X. and Liu, R.H. (2003) Antioxidant Activity of Apple Peels. Journal of Agricultural and Food Chemistry, 51, 609-614. https://doi.org/10.1021/jf020782a

[20] Statistica Software (2019). https://www.tibco.com/products/data-science

[21] Khanizadeh, S., Tsao, R., Rekika, D., Yang, R., Charles, M.T. and Rupasinghe, H.P.V. (2008) Polyphenol Composition and Total Antioxidant Capacity of Selected Apple Genotypes for Processing. Journal of Food Composition and Analysis, 21, 396-401. https://doi.org/10.1016/j.jfca.2008.03.004

[22] Mainla, L., Moor, U., Karp, K. and Pussa, T. (2011) The Effect of Genotype and Rootstock on Polyphenol Composition of Selected Apple Cultivars in Estonia. Zemdirbyste-Agriculture, 98, 63-70.

[23] Murtic, S., Civic, H., Duric, M., Sekularac, G., Kojovic, R., Kulina, M. and Krsmanovic, M. (2012) Foliar Nutrition in Apple Production. African Journal of Biotechnology, 11, 10462-10468. https://doi.org/10.5897/AJB12.477

[24] Renard, C.M.G.C., Dupont, N. and Guillermin, P. (2007) Concentrations and Characteristics of Procyanidins and Other Phenolics in Apples during Fruit Growth. Phytochemistry, 68, 1128-1138. https://doi.org/10.1016/j.phytochem.2007.02.012

[25] Cripps, J.E.L., Richards, L.A. and Mairata, A.M. (1993) 'Pink Lady' Apple. HortScience, 28, 1057. https://doi.org/10.21273/HORTSCI.28.10.1057

[26] Whale, S.K. and Singh, Z. (2007) Endogenous Ethylene and Color Development in the Skin of 'Pink Lady' Apple. Journal of the American Society for Horticultural Science, 132, 20-28. https://doi.org/10.21273/JASHS.132.1.20

[27] Lister, C.E., Lancaster, J.E., Sutton, K.H. and Walker, J.R.L. (1994) Developmental Changes in the Concentration and Composition of Flavonoids in Skin of a Red and a Green Apple Cultivar. Journal of the Science of Food and Agriculture, 64, 155-161. https://doi.org/10.1002/jsfa.2740640204

[28] Hamauzu, Y., Iijima, E. and Banno, K. (1999) Changes in Catechin and Procyanidin Contents during Fruit Development of Two Apple Cultivars. Journal of Japanese Society of Horticultural Science, 68, 1184-1193. https://doi.org/10.2503/jjshs.68.1184

[29] Awad, M.A., De Jager, A., Van Der Plas, L.H.W. and Van Der Krol, A.R. (2001) Flavonoid and Chlorogenic Acid Changes in Skin of 'Elstar' and 'Jonagold' Apples during Development and Ripening. Scientia Horticulturae, 90, 69-83. https://doi.org/10.1016/S0304-4238(00)00255-7

[30] Kondo, S., Tsuda, K., Muto, N. and Ueda, J.E. (2002) Antioxidative Activity of Apple Skin or Flesh Extracts Associated with Fruit Development on Selected Apple Cultivars. Scientia Horticulturae, 96, 177-185. https://doi.org/10.1016/S0304-4238(02)00127-9

[31] Moyle, C.W.A. (2011) Polyphenols in Apples and Their Interactions with Vascular Endothelial Cells. PhD Dissertation, University of East Anglia, Norwich.

[32] Mehrabani, L.V. and Hassanpouraghdam, B.M. (2012) Developmental Variation of Phenolic Compounds in Fruit Tissue of Two Apple Cultivars. Acta Scientiarum Polonorum, 11, 259-264.

[33] Hamadziripi, E.T. (2012) The Effect of Canopy Position on the Fruit Quality and Consumer Preference of Apples. MSc Thesis, Stellenbosch University, Stellenbosch. 
[34] Gupta, C.U., Jame, W.Y., Campbell, A.C., Leyshon, J.A. and Nicholaichuk, W. (1985) Boron Toxicity and Deficiency: A Review. Canadian Journal of Soil Science, 65, 381-409. https://doi.org/10.4141/cjss85-044

[35] Nable, O.R., Banuelos, S.G. and Paull, G.J. (1997) Boron Toxicity. Plant and Soil, 193, 181-198. https://doi.org/10.1023/A:1004272227886

[36] Feng, F., Li, M., Ma, F. and Cheng, L. (2014) The Effects of Bagging and Debagging on External Fruit Quality, Metabolites, and the Expression of Anthocyanin Biosynthetic Genes in 'Jonagold' Apple (Malus domestica Borkh.). Scientia Horticulturae, 165, 123-131. https://doi.org/10.1016/j.scienta.2013.11.008

[37] Feng, F., Li, M., Ma, F. and Cheng, L. (2014) Effects of Location within the Tree Canopy on Carbohydrates, Organic Acids, Amino Acids and Phenolic Compounds in the Fruit Peel and Flesh from Three Apple (Malus $\times$ domestica) Cultivars. Horticulture Research, 1, 14019. https://doi.org/10.1038/hortres.2014.19

[38] De Paepe, D., Valkenborg, D., Noten, B., Servaes, K., Diels, L., De Loose, M., Van Droogenbroeck, B. and Voorspoels, S. (2015) Variability of the Phenolic Profiles in the Fruits from Old, Recent and New Apple Cultivars Cultivated in Belgium. Metabolomics, 11, 739-752. https://doi.org/10.1007/s11306-014-0730-2

[39] Falcone Ferreyra, M.L., Rius, S.P. and Casati, P. (2012) Flavonoids: Biosynthesis, Biological Functions, and Biotechnological Applications. Frontiers in Plant Science, 3, 222. https://doi.org/10.3389/fpls.2012.00222

[40] Liu, M., Li, X., Liu, Y. and Cao, B. (2013) Regulation of Flavanone 3-Hydroxylase Gene Involved in the Flavonoid Biosynthesis Pathway in Response to UV-B Radiation and Drought Stress in the Desert Plant, Reaumuria soongorica. Plant Physiology and Biochemistry, 73, 161-167. https://doi.org/10.1016/j.plaphy.2013.09.016

[41] Song, X., Diao, J., Ji, J., Wang, G., Guan, C., Jin, C. and Wang, Y. (2016) Molecular Cloning and Identification of a Flavanone 3-Hydroxylase Gene from Lycium chinense, and Its Overexpression Enhances Drought Stress in Tobacco. Plant Physiology and Biochemistry, 98, 89-100. https://doi.org/10.1016/j.plaphy.2015.11.011

[42] Agati, G., Azzarello, E., Pollastri, S. and Tattini, M. (2012) Flavonoids as Antioxidants in Plants: Location and Functional Significance. Plant Science, 196, 67-76. https://doi.org/10.1016/j.plantsci.2012.07.014

[43] Agati, G., Brunetti, C., Ferdinando, M.D., Ferrini, F., Pollastri, S. and Tattini, M. (2013) Functional Roles of Flavonoids in Photoprotection: New Evidence, Lessons from the Past. Plant Physiology and Biochemistry, 72, 35-45. https://doi.org/10.1016/j.plaphy.2013.03.014

[44] Merzlyak, M.N., Solovchenko, A.E., Smagin, A.I. and Gitelson, A.A. (2005) Apple Flavonols during Fruit Adaptation to Solar Radiation: Spectral Features and Technique for Non-Destructive Assessment. Journal of Plant Physiology, 162, 151-160. https://doi.org/10.1016/j.jplph.2004.07.002 